\title{
Elastic heat exchanger in Stirling cycle machines
}

\author{
Y. Kobayashi ${ }^{1}$, M. Matsuo ${ }^{2}$, N. Isshiki ${ }^{3}$ \& W. Ishida ${ }^{4}$ \\ ${ }^{1}$ Tsuchiura Technical High-school, Japan \\ ${ }^{2}$ Saitama University, Japan \\ ${ }^{3}$ Tokyo Institute of Technology, Japan \\ ${ }^{4}$ IDEC Ltd., Japan
}

\begin{abstract}
The Stirling cycle machine technology is expected to be one of the solutions for preventing global warming. The machines achieving high thermal efficiency use hydrogen or helium as a working fluid. The average pressure in the machines is usually raised to $10 \mathrm{MPa}$ or more. These light gases need a precise sealing mechanism so that no leakage and minimum friction loss are achieved. Therefore, the use of light gases induces high cost, and makes maintenance difficult. Of course, any gas can be used in the Stirling cycle. Air was used in the 19th century. However, when air is used as a working fluid, the heat transfer in the engine becomes minimal. Consequently, the thermal efficiency falls in the engines and the COP falls in the refrigerators or heat pumps. In this paper, a method for heat transfer enhancement between the working gas and the walls of an air-charged Stirling cycle machine is attempted by installing a heat exchanger that changes shape with elasticity in the working space. By applying the method to a small engine and refrigerator charged with atmospheric air, the enhancement in heat transfer is confirmed.
\end{abstract}

Keywords: Stirling cycle machines, air-charged engine, refrigeration, heat transfer enhancement, temperature drops, elastic heat exchanger.

\section{Introduction}

Air has some advantages as a working fluid. It is abundant and available all over the world. It is less difficult to seal than the light gases. Therefore, an air charged machine can be supplied at low price and will be easy to maintain. That is 
suitable for a small generating engine using biomass or solar power in a remote place for example. Many studies on the air-charged refrigerator have also been done. For example, Ohtaka [1] reported an experimental result that a refrigerator filled with nitrogen had the performance which was not inferiority compared with helium at a slow rotation speed.

Air has two disadvantages as a working fluid: a decrease of heat transfer and an increase of pressure loss. Gas temperatures of the cold part and hot part determine the theoretical thermal efficiency of a Stirling cycle. Therefore, the temperature drop at the walls of a heater and cooler of the Stirling machine causes a fall in the efficiency. Many Stirling machines use some tubes as their heat exchanger.

Walker et al [2] compared the heat transfer per unit pressure drop for air and helium by an elementary thermo fluid analysis about a single tube:

$$
\frac{(\dot{Q} / \Delta P)_{\text {helium }}}{(\dot{Q} / \Delta P)_{\text {air }}}=\left(\frac{K_{H}}{K_{A}}\right)^{2 / 3}\left(\frac{M_{A}}{M_{H}}\right)^{1 / 5}\left(\frac{S_{H}}{S_{A}}\right)^{1 / 3}\left(\frac{\mu_{A}}{\mu_{H}}\right)^{1 / 2}
$$

where $K=$ thermal conductivity, $M=$ molecular weight, $S=$ specific heat and $\mu=$ viscosity. The eqn (1) gives the value 7.4 , and the same calculation for air and hydrogen gives a value over 12. It is observable that these values are independent to a change in length and thickness of the pipe. In order to enhance the heat transfer, the method of increasing the surface area of the tubes is usually adopted in the light gas engines. However, this method is accompanied by an increase in dead volume and pressure loss. The size and shape of the pipe that is profitable to only an air engine will not be expected.

As heat exchangers other than tubes, the cap-type heater or cooler is used in some engines or refrigerators. It has a simpler structure, and makes both dead volume and pressure loss a minimum, although the area for heat transfer is not enough.

In order to enhance the heat transfer and not to increase the dead volume and the pressure loss, we have proposed the elastic heat exchanger attached on the surface of the expansion piston in the cap-type air-charged engine and refrigerator. The effect of the heat exchanger is experimentally examined in this paper.

\section{The elastic heat exchanger}

As shown in fig. 1, the elastic heat exchanger (EHE) is attached to the end of the expansion piston. EHE consists of a wire mesh and a plate with elasticity. As shown in fig. 2, at the top dead centre of expansion piston, EHE is pushed against the cap wall and heat is supplied. Then, EHE is separated from the wall and contacts to the working fluid.

In order to secure the contact time, the elasticity of the plate is required. Sufficient heat capacity and surface area of the wire mesh are needed for heat transfer into the fluid. 
Various forms can be considered for the EHE, such as a metal plate with springs, coils, sponge, etc. In the first measurement, a plate made from stainless fibre has been chosen as an elastic plate for the heat resistance.

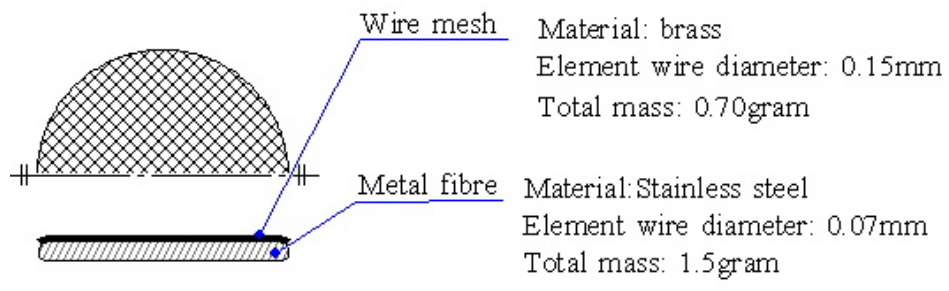

Figure 1: Tested EHE.

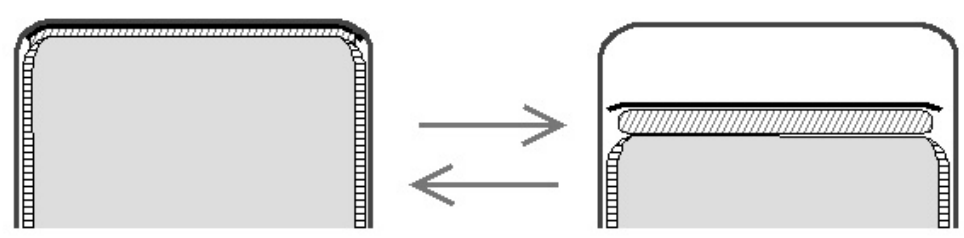

Figure 2: EHE contacting with the cap wall.
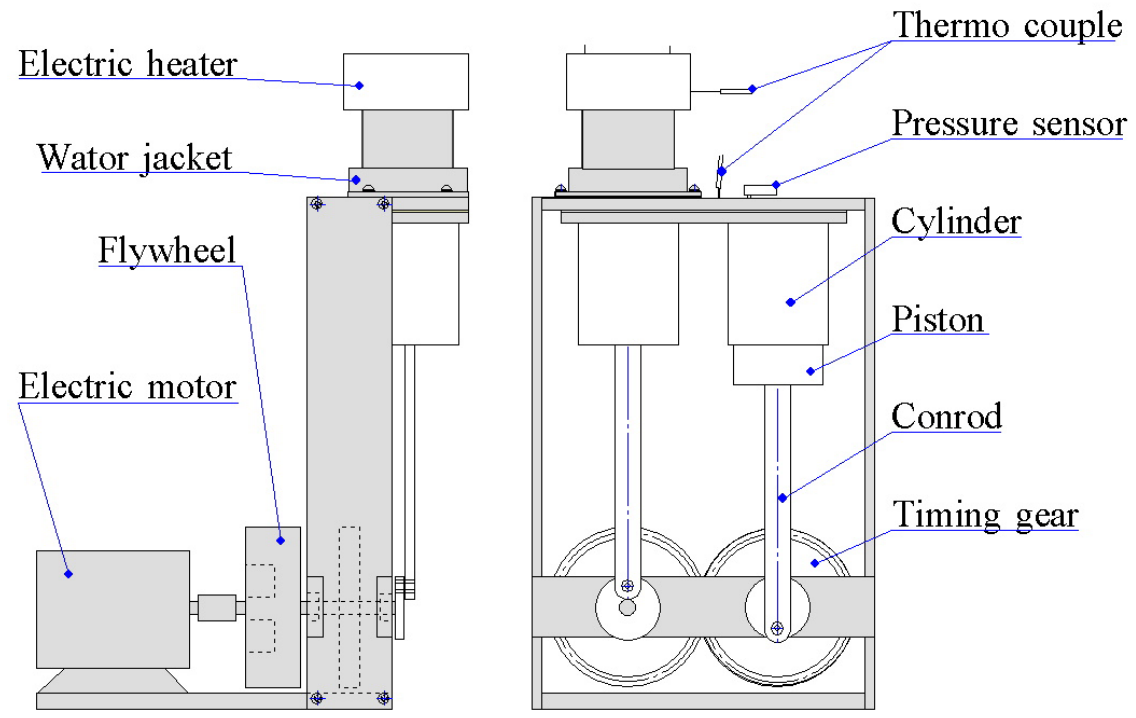

Figure 3: Schematic figure of measurement apparatus. 


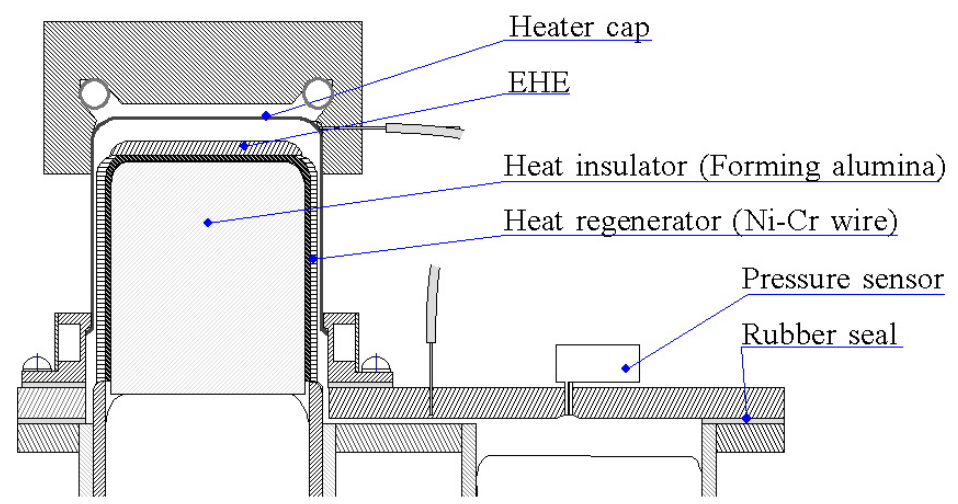

Figure 4: Detail figure of volume arrangement.

Table 1: $\quad$ Volumes for calculation.

\begin{tabular}{|l|r|}
\hline Swept volume of the expansion piston & $28.6 \mathrm{cc}$ \\
\hline Swept volume of the compression piston & $28.6 \mathrm{cc}$ \\
\hline Volume of regenerator space & $10.4 \mathrm{cc}$ \\
\hline Dead volume in the expansion cylinder & $4.2 \mathrm{cc}$ \\
\hline Dead volume in the compression cylinder & $7.5 \mathrm{cc}$ \\
\hline
\end{tabular}

\section{Measurement of the pressure ratio}

A schematic figure of measurement apparatus is shown in fig. 3. This is a twopiston type Stirling engine that has a phase angle of 180 degrees. The total volume of working fluid is kept constant. Two crankshafts interlock by two timing gears. One of the shafts is connected to a geared electric motor. The temperature sensors are attached on the wall of the heat-cap and the cooler plate. The gaseous pressure of the compression space is measured with a silicon piezoresistive pressure sensor.

The detailed figure of the space arrangement is shown in fig. 4. The heat-cap is made from $0.3 \mathrm{~mm}$ thick stainless steel. The electric heater is mounted with a foaming alumina block on the heat-cap. The metal wire is coiled around the piston sidewall as a heat regenerator. The cooler plate consists of two aluminum plates and a silicone rubber plate. The central part of the rubber plate is cut off belt-like and the working fluid flows along the crevice.

The results from the operation at rotation speeds of $5 \mathrm{~Hz}$ and $10 \mathrm{~Hz}$ are shown in fig. 5. By installing EHE, the pressure ratio $P_{\max } / P_{\min }$ increased. This means that the temperature difference between the wall and the fluid decreased. The 
improvement of the heat transfer has brought the improvement of the gas temperature ratio by about 0.08 .

The effect of EHE decreases a little at $10 \mathrm{~Hz}$. As the cause of this, the contact time of EHE to the heat-cap wall can be considered. The increase of rotation speed shortens the contact time length. The heat replenishment with EHE by the conduction of heat might be insufficient. For preventing this, it may be effective in increasing the thickness of sponge. Considering this from a different viewpoint, the discontinuous movement of the piston could make EHE more effective [4].
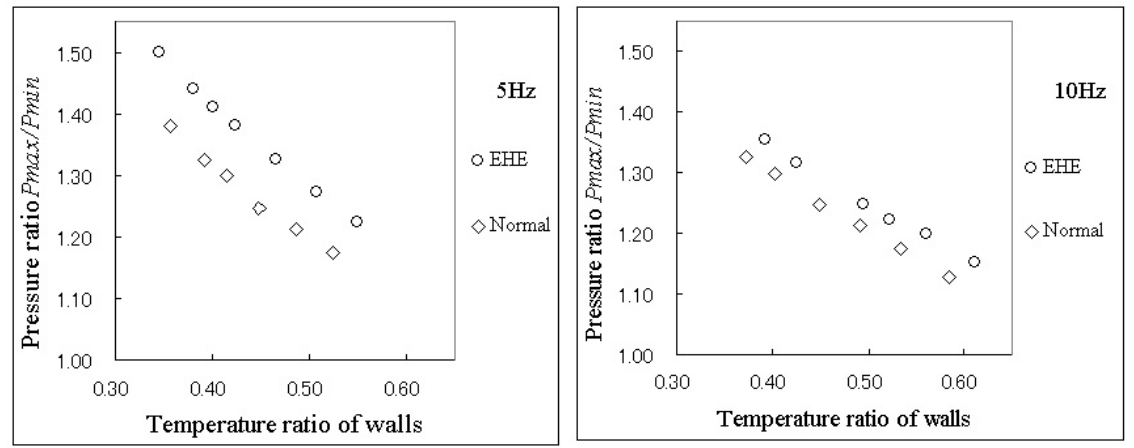

Figure 5: $\quad$ Pressure ratio versus temperature ratio of walls.

\section{Calculation for the gas temperature ratio}

Measuring the temperature of the working gas of an actual expansion space directly and correctly is accompanied by difficulties, such as heat capacity and conductivity of sensor and change of the flow velocity. If some appropriate conditions are accepted, the relations between the temperature ratio and the pressure ratio are easily provided.

In the ideal isothermal model calculation called the Schmidt theory [3], the following conditions are assumed.

(a) Volumes of the expansion space and the compression space change are sinusoidal.

(b) There is no pressure loss and there is no internal pressure difference. The moment pressure is $P$.

(c) The equation of state for a perfect gas applies.

(d) The temperatures of gas are homogeneous in each of the spaces.

(e) The gas temperature in the expansion space is constant $T_{E}$ and the gas temperature in the cold space is constant $T_{C}$ during a cycle.

For simplification, we assume that the total dead volume $V_{D}$ includes the volumes of the expansion cylinder and the compression cylinder as shown in table 2. 
Table 2: $\quad$ Space partitions.

\begin{tabular}{|l|l|l|}
\hline Spaces & Volume & Temperature \\
\hline Expansion space & $V_{E}$ & $T_{E}$ \\
\hline $\begin{array}{l}\text { Regenerator, cooler } \\
\text { and other dead spaces }\end{array}$ & $V_{D}$ & $\frac{T_{E}+T_{C}}{2}$ \\
\hline Compression space & $V_{C}$ & $T_{C}$ \\
\hline
\end{tabular}

By the assumption (a), the volumes of the expansion space and the compression space are expressed as a function of the crank angle $\theta$

$$
V_{E}=V_{S E} \frac{1-\cos \theta}{2}, \quad V_{C}=V_{S C} \frac{1-\cos (\theta-\beta)}{2} \text {. }
$$

where $\beta$ is the crank angle difference of two pistons. The mass conservation law and the equation of state for the perfect gas draw the moment gas pressure $P$ as a function of $\theta$.

$$
\begin{gathered}
P=P_{\text {mean }} \frac{\sqrt{1-\delta^{2}}}{1-\delta \cos (\theta-\phi)}, \quad \phi=\tan ^{-1}\left(\frac{\kappa \sin \beta}{\tau+\kappa \cos \beta}\right), \\
\delta=\sqrt{\kappa^{2}+\tau^{2}+2 \kappa \tau \cos \beta} /\left(\kappa+\tau+\frac{4 \tau \kappa_{D}}{1+\tau}\right)
\end{gathered}
$$

Here, $P_{\text {mean }}$ is the average of $P$ on $\theta, \kappa$ is the swept volume ratio, $\kappa_{D}$ is the dead volume ratio, and $\tau$ is the gas temperature ratio.

$$
\kappa=\frac{V_{S C}}{V_{S E}}, \quad \kappa_{D}=\frac{V_{D}}{V_{S E}}, \tau=\frac{T_{C}}{T_{E}}
$$

By using eqn (2), the maximum pressure ratio is given as

$$
\frac{P_{M A X}}{P_{M I N}}=\frac{1+\delta}{1-\delta} \text {. }
$$

Then, if the geometrical values $\kappa, \kappa_{D}$ and $\beta$ are given, the relations between the pressure ratio and the gas temperature ratio are provided as shown in fig. 6 for example. 


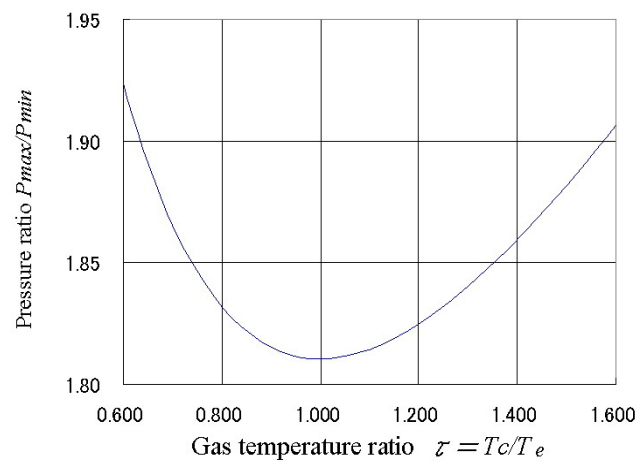

Figure 6: The gas temperature ratio as a function of the pressure ratio $(\kappa=1$,

$$
\left.\kappa_{D=0.989,} \beta=110 \mathrm{deg}\right) \text {. }
$$

\section{Adopting EHE to refrigerator}

Adopting EHE to a refrigerator is to examine a range of $\tau \geq 1$ in fig. 6 . The examined refrigerator is the apparatus shown in fig. 3. The phase angle difference is set at 110 degrees. EHE has been changed as shown in fig. 7. Adjusting the power of the electric heater and keeping the temperature of the cold-cap constant, the pressure change is measured.

The relation between the temperature ratio of walls and the pressure ratio is shown in Fig. 8. The whole values of pressure ratio at $4 \mathrm{~Hz}$ are lower. It is considered as the leakage of working fluid through the piston seal.

The temperature change of the cold head when the refrigerator operates with no load is shown in the figure on the left in fig. 9. The change of cooling capacity is shown in the figure on the right in fig. 9. In this figure, the cooling capacity is expressed by the ratio to the value of the normal $4 \mathrm{~Hz}$ operation at $0^{\circ} \mathrm{C}$. The heat capacity of this apparatus is about 1 watt at $0^{\circ} \mathrm{C}$. The cooling capacity of this apparatus is 1 watt or less for the temperature difference of $10 \mathrm{~K}$ at speed $4 \mathrm{~Hz}$.

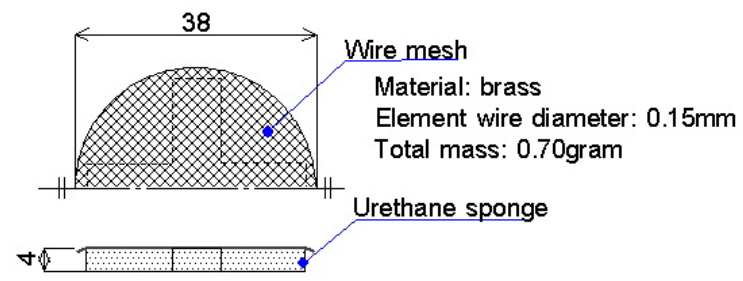

Figure 7: $\quad$ EHE for a refrigerator. 


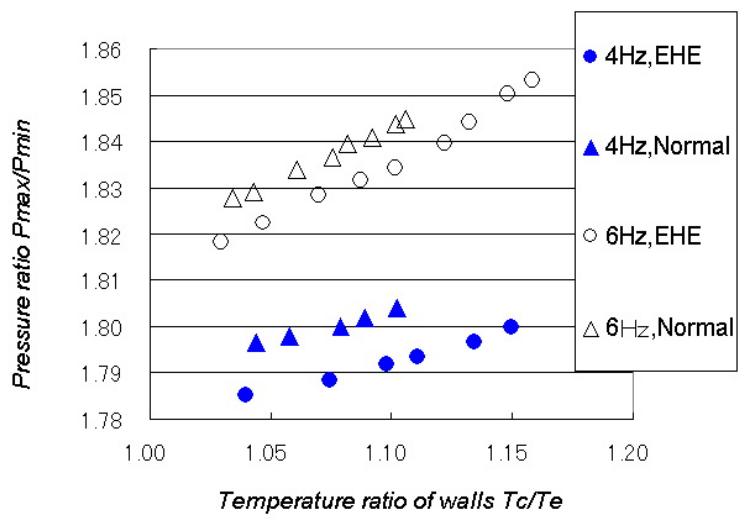

Figure 8: $\quad$ Pressure ratio versus temperature ratio of walls.
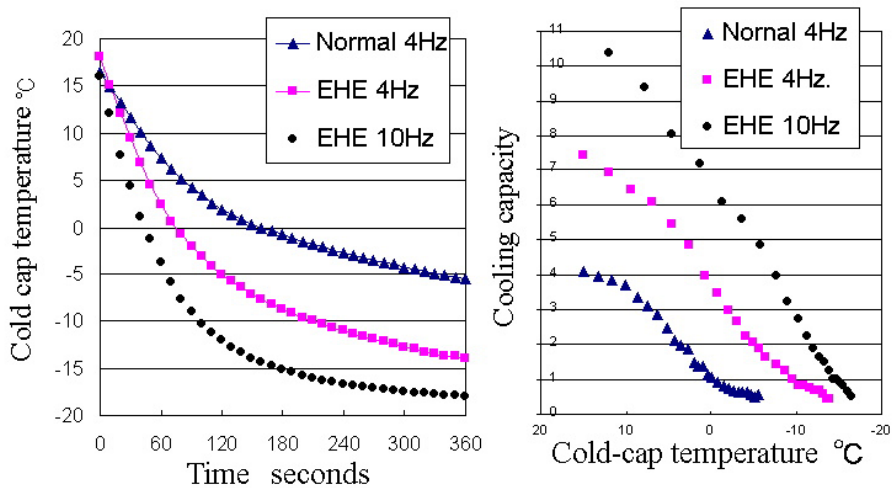

Figure 9: Cold cap change and cooling capacity.

\section{Conclusion}

\subsection{The effectiveness of EHE}

It has been confirmed by the measurement that EHE enhances the heat transfer in the cap-type air-charged Stirling cycle machines. EHE seems to be more effective at low speeds of rotation. The temperature ratio in the engine has been improved by about 0.1 at the maximum. The cooling capacity of the examined refrigerator has increased by 2 to 3 times.

\subsection{The gas temperature ratio estimation}

The gas temperature ratio can be easily obtained from measurement of the pressure ratio by using the result of the Schmidt calculation. In the measurement of a slow speed machine, the pressure ratio tends to become slightly lower than 
the theoretical value. The reason for this is considered to be the leakage of gas through seals in the examined machine.

\subsection{Performance of EHE}

In the high-speed rotation, the lack of the heat replenishment is estimated. The heat replenishment from the cap wall doesn't increase only by an increase in the heat capacity of EHE. There is a limit in increasing the contact time in the sinusoidal movement of the piston. The piston having a discontinuous movement might make EHE more effective.

\section{References}

[1] Otaka, T, Study of Performance Characteristics on a Small Size Stirling Refrigerator, Transactions of the Japan Society of Mechanical Engineers. B, (ISSN 03875016), pp 1049-1057, 2001

[2] Walker, G. et al, The Stirling Alternative, Gordon and Breach Publishers Inc., pp 85-87, 1994

[3] G.Schmidt, Theore der Lehmannschen Calorischen Maschine, Zeitschrift des Vereines deutscher Ingenieure, 1871

[4] Kobayashi, Y, Model Stirling Cooler with Colliding Displacer", Proc of $9^{\text {th }}$ Symposium on Stirlling Cycle( ISSN 09189238), pp 125-126, 2005 